\title{
Is a Single Molecule Sufficient to Determine the Internal Charge Trapping Energy in Crystalline Organic
}

\section{Semiconductors?}

Lingzhi Tu and Hongguang Liu $u^{\mathrm{a}, \mathrm{b}, *}$

${ }^{a}$ College of Chemistry and Materials Science, Jinan University, 601 Huang-Pu Avenue West, Guangzhou 510632, China.

${ }^{\mathrm{b}}$ Guangdong Provincial Key Laboratory of Functional Supramolecular Coordination Materials and Applications, Jinan University, 601 Huang-Pu Avenue West, Guangzhou 510632, China.

*E-mail: hongguang_liu@jnu.edu.cn

\section{Contents}

Isolated and embedded molecular structures for 15-21 (Figure S1)

Comparison on the computed hole mobilities and the experimental data for some representatives (Table S1)

Reference in Table S1 


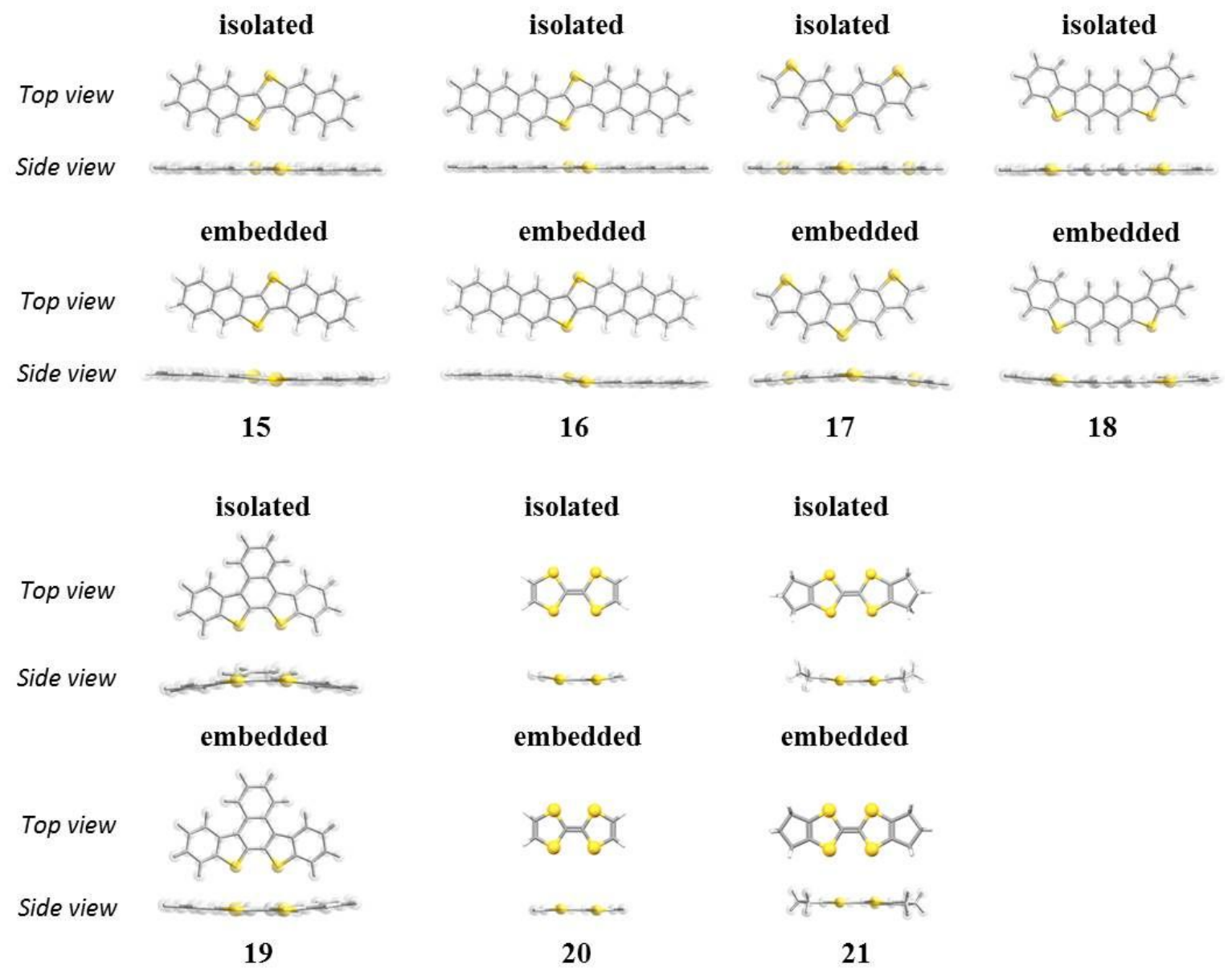

Figure S1. Isolated and embedded molecular structures for 15-21. 
Table S1. Comparison on the computed hole mobilities and the experimental data for some representatives.

\begin{tabular}{|c|c|c|c|c|c|}
\hline molecule & $\begin{array}{c}\lambda_{\text {iso_NFP }} / \lambda_{\text {emb_NFP }} \\
(\mathrm{meV})\end{array}$ & $\begin{array}{c}\mathbf{V}_{\max } \\
(\mathrm{meV})\end{array}$ & $\begin{array}{l}\boldsymbol{\mu}_{\text {computational }} \\
\left(\mathrm{cm}^{2} \mathrm{~V}^{-1} \mathrm{~s}^{-1}\right)\end{array}$ & $\begin{array}{l}\boldsymbol{\mu}_{\text {experimental }} \\
\left(\mathrm{cm}^{2} \mathrm{~V}^{-1} \mathrm{~s}^{-1}\right)\end{array}$ & Ref \\
\hline 16 & $85 / 102$ & 95.2 & $10.5 / 8.1$ & 3 & [1] \\
\hline 17 & $117 / 143$ & 45.6 & $2.35 / 1.65$ & 0.011 & [2] \\
\hline 20 & $330 / 278$ & 111.1 & $0.46 / 0.82$ & 1.2 & [3] \\
\hline 25 & $267 / 198$ & 31.9 & $0.36 / 0.81$ & 3.7 & [4] \\
\hline 42 & $305 / 228$ & 22.5 & $0.035 / 0.085$ & 0.2 & [5] \\
\hline 44 & $314 / 216$ & 13.3 & $0.015 / 0.048$ & 0.09 & [6] \\
\hline
\end{tabular}

$\mathrm{V}_{\max }$ is the largest transfer integral (site-energy corrected) computed from the crystal structure at the B3LYP/6-31G(d,p) level. 
[1] Niimi, K.; Shinamura, S.; Osaka, I.; Miyazaki, E.; Takimiya, K. Dianthra[2,3-B:2',3'-F]Thieno[3,2-B]Thiophene (DATT): Synthesis, Characterization, and Fet Characteristics of New Pi-Extended Heteroarene with Eight Fused Aromatic Rings. J. Am. Chem. Soc. 2011, 133, 8732-8739.

[2] Wex, B.; Kaafarani, B. R.; Schroeder, R.; Majewski, L. A.; Burckel, P.; Grell, M.; Neckers, D. C. New Organic Semiconductors and Their Device Performance as a Function of Thiophene Orientation. J. Mater. Chem. 2006, 16, 1121-1124.

[3] Jiang, H.; Yang, X.; Cui, Z.; Liu, Y.; Li, H.; Hu, W.; Liu, Y.; Zhu, D. Phase Dependence of Single Crystalline Transistors of Tetrathiafulvalene. Appl. Phys. Lett. 2007, 91, 123505.

[4] Tripathi, A. K.; Heinrich, M.; Siegrist, T.; Pflaum, J. Growth and Electronic Transport in 9,10-Diphenylanthracene Single Crystals-An Organic Semiconductor of High Electron and Hole Mobility. Adv. Mater. 2007, 19, 2097-2101.

[5] Chen, M. C.; Vegiraju, S.; Huang, C. M.; Huang, P. Y.; Prabakaran, K.; Yau, S. L.; Chen, W. C.; Peng, W. T.; Chao, I.; Kim, C.; Tao, Y. T. Asymmetric Fused Thiophenes for Field-Effect Transistors: Crystal Structure-Film Microstructure-Transistor Performance Correlations. $J$. Mater. Chem. C 2014, 2, 8892-8902.

[6] Noh, Y. Y.; Azumi, R.; Goto, M.; Jung, B. J.; Lim, E.; Shim, H. K.; Yoshida, Y.; Yase, K.; Kim, D. Y. Organic Field Effect Transistors Based on Biphenyl, Fluorene End-Capped Fused Bithiophene Oligomers. Chem. Mater. 2005, 17, 3861-3870. 